\title{
ROTATIONAL FRICTION COEFFICIENT OF A PERMEABLE CYLINDER IN A VISCOUS FLUID
}

\author{
F.W. WIEGEL \\ Department of Applied Physics, Twente University of Technology, 7500 AE Enschede, The Netherlands
}

Received 20 September 1978

An exact expression is derived for the rotational friction coefficient of a cylinder of infinite length and constant permeability immersed in an incompressible viscous fluid. An asymptotic expression for the translational friction coefficient of a permeable cylinder moving in a sheet of viscous fluid embedded on both sides in a fluid of much lower viscosity is also given.

In this letter we consider a porous cylinder of infinite length and uniform permeability immersed in an incompressible fluid (viscosity $\eta$ ) and rotating with a constant angular velocity $\omega_{0}$ around its axis. The problem is to calculate the rotational friction coefficient $f_{\mathrm{R}}$, which is defined as the ratio $T / \omega_{0}$, where $T$ denotes the total torque of the forces per unit of length which the cylinder exerts on the fluid.

This problem is relevant to the rotational diffusion of a patch of cross-linked proteins in a cell membrane [1]. This rotational friction coefficient has never been calculated; the analogous problem for a sphere of uniform permeability was solved only a few years ago by Felderhof and Deutch [2].

Consider a cartesian system of coordinates $(x, y, z)$ with the $z$-axis along the axis of the cylinder. (Presently, we shall also use cylindrical coordinates $(r, \phi, z)$.) Let $\boldsymbol{V}$ and $P$ denote the average local velocity and pressure of the fluid and $U$ the local velocity of the cylinder. The fluid flow has to be solved from the DebyeBrinkman-Bueche equation:

$-\nabla P+\eta \Delta V-(\eta / k)(\boldsymbol{V}-\boldsymbol{U})=0$,

together with the incompressibility equation:

$\operatorname{div} V=0$.

In this note we only consider the case of a uniform cylinder for which the permeability $k(r)=k_{0}$ if $r<a$, $k(r)=\infty$ if $r>a ; a$ denotes the radius of the cylinder. A microscopic derivation of eq. (1) has been given by
Felderhof and Deutch [2]; and a macroscopic derivation was given by Wiegel and Mijnlieff [3]. Applications of the Debye-Brinkman-Bueche equation to the flow of a solvent through a polymer coil are also found in refs. [4-10].

The $x$ and $y$ components of $U$ are:

$U_{x}=-\omega_{0} r \sin \phi ; \quad U_{y}=+\omega_{0} r \cos \phi$.

For the pressure and the velocity we make the ansatz:

$P=$ constant

$V_{x}=-V(r) \sin \phi ; \quad V_{y}=+V(r) \cos \phi$,

where $V(r)$ denotes an unknown function - the magnitude of the velocity - which has cylindrical symmetry. Upon substitution of eqs. (4) and (5) into eqs. (1) and (2) one finds that all equations are satisfied provided $V(r)$ is the solution of the ordinary differential equation:

$V^{\prime \prime}+\frac{1}{r} V^{\prime}-\frac{1}{r^{2}} V=\left\{\begin{array}{ll}V / k_{0}-\omega_{0} r / k_{0}, & 0<r<a, \\ 0, & a<r .\end{array}(6 \mathrm{a}, \mathrm{b})\right.$

The boundary conditions are: (i) $V(0)$ should be finite; (ii) $V(\infty)=0$; (iii) $V$ and $V^{\prime}$ should be continuous at $r=a$.

The solution of this differential equation is straightforward and one finds:

$V(r)= \begin{cases}\omega_{0} r+B I_{1}\left(r / \sqrt{k_{0}}\right), & 0<r<a, \\ A / r, & a<r,\end{cases}$ 
with:

$A=\omega_{0} a^{2} I_{2}(\sigma) / I_{0}(\sigma)$,

$B=-2 \omega_{0} a / \sigma I_{0}(\sigma)$.

In these formulae the dimensionless parameter $\sigma$ $\equiv a / \sqrt{k_{0}}$ is the ratio of the radius of the cylinder and the distance $\sqrt{k_{0}}$ over which the fluid flow effectively penetrates the cylinder. The $I_{\nu}(\sigma)$ denote the modified Bessel functions.

The torque of the forces which the cylinder exerts on the fluid, per unit of length, equals:

$T=-2 \pi B \frac{\eta}{k_{0}} \int_{0}^{a} r^{2} I_{1}\left(r / \sqrt{k_{0}}\right) \mathrm{d} r=4 \pi \eta \omega_{0} a^{2} \frac{I_{2}(\sigma)}{I_{0}(\sigma)}$

Hence the rotational friction coefficient per unit of length is given by:

$f_{\mathrm{R}}=4 \pi \eta a^{2} I_{2}(\sigma) / I_{0}(\sigma)$.

In the limit $\sigma \rightarrow \infty$ the cylinder becomes impermeable and one recovers the trivial result $f_{\mathrm{R}}=4 \pi \eta a^{2}$ which holds for a hard cylinder. If, on the other hand, $\sigma \ll 1$ the expression (11) simplifies to $f_{\mathrm{R}}=\frac{1}{2} \pi \eta a^{2} \sigma^{2}$, as it should. Using tables of the modified Bessel functions [11], we have tabulated in table 1 the correction factor due to the finite permeability of the cylinder.

For the sake of comple teness, we also give the translational friction coefficient (force per unit relative velocity) of a permeable cylinder of height $h$ constrained to move in a sheet of fluid with viscosity $\eta$. The sheet has thickness $h$ and is embedded on both sides in another fluid of a much lower viscosity $\eta^{\prime}$. The translational friction coefficient is given by:

$f_{\mathrm{T}}=4 \pi \eta h\left\{-\gamma+\ln \left(h \eta / a \eta^{\prime}\right)+\frac{2}{\sigma^{2}}+\frac{I_{0}(\sigma)}{\sigma I_{1}(\sigma)}\right\}^{-1}$,

provided $\left(h \eta / a \eta^{\prime}\right) \gg 1$. In this formula $\gamma=0.5772 \mathrm{de}-$ notes Euler's constant. The derivation of eq. (12) will
Table 1

The second column gives the correction factor to the hard disk rotational friction coefficient due to finite permeability, eq. (11); the third column gives the correction term to the inverse of the hard disk translational friction coefficient, eq. (12).

\begin{tabular}{rll}
\hline$\sigma$ & $I_{2}(\sigma) / I_{0}(\sigma)$ & $\left(2 / \sigma^{2}\right)+I_{0}(\sigma) / \sigma I_{1}(\sigma)$ \\
\hline 0 & 0 & $\infty$ \\
1 & 0.1072 & 4.2402 \\
2 & 0.3022 & 1.2166 \\
3 & 0.4600 & 0.6337 \\
4 & 0.5682 & 0.4145 \\
5 & 0.6426 & 0.3039 \\
6 & 0.6958 & 0.2382 \\
7 & 0.7355 & 0.1952 \\
8 & 0.7662 & 0.1649 \\
9 & 0.7905 & 0.1426 \\
10 & 0.8103 & 0.1254 \\
\hline
\end{tabular}

be given elsewhere [12]. The correction term $\left(2 / \sigma^{2}\right)$ $+I_{0}(\sigma) / \sigma I_{1}(\sigma)$ has also been tabulated in table 1 .

\section{References}

[1] F.W. Wiegel, Hydrodynamics of a permeable patch in the fluid membrane, to be published.

[2] B.U. Felderhof and J.M. Deutch, J. Chem. Phys. 62 (1975) 2391.

[3] F.W. Wiegel and P.F. Mijnlieff, Physica 85A (1976) 207.

[4] G. Ooms, P.F. Mijnlieff and H.L. Beckers, J. Chem. Phys. 53 (1970) 4123.

[5] B.U. Felderhof and J.M. Deutch, J. Chem. Phys. 62 (1975) 2398.

[6] B.U. Felderhof, Physica 80A (1975) 63.

[7] B.U. Felderhof, Physica 80A (1975) 172.

[8] F.W. Wiegel and P.F. Mijnlieff, Polymer 18 (1977) 636.

[9] F.W. Wiegel and P.F. Mijnlieff, Physica 89A (1977) 385.

[10] P.F. Mijnlieff and F.W. Wiegel, J. Polym. Sci. Phys. Ed. 16 (1978) 245.

[11] M. Abramowitz and I.A. Stegun, Handbook of Mathematical functions (Dover, New York, 1968).

[12] F.W. Wiegel, Translational friction coefficient of a permeable cylinder in a sheet of viscous fluid, to be published. 